\title{
El problema social de la educación virtual universitaria en tiempos de pandemia, Perú
}

Social Issues in virtual higher education during pandemic's time in Peru

O problema social da educação virtual universitária em tempos de pandemia, Peru

\author{
Jesús Wiliam Huanca-Arohuanca \\ Universidad Nacional de San Agustín de Arequipa \\ Arequipa, Perú \\ jhuancaar@unsa.edu.pe \\ (1) https://orcid.org/0000-0002-7353-1166 \\ Felipe Supo-Condori \\ Universidad Nacional del Altiplano \\ Puno, Perú \\ fsupo@unap.edu.pe \\ (D) https://orcid.org/0000-0002-5007-9779 \\ Reynaldo Sucari Leon \\ Universidad Nacional Autónoma de Huanta \\ Ayacucho, Perú \\ rsucari@unah.edu.pe \\ (DD) https://orcid.org/0000-0001-7824-5292 \\ Luis Alberto Supo Quispe \\ Universidad Nacional del Altiplano \\ Puno, Perú \\ ingluisalberto83@gmail.com \\ (iD) https://orcid.org/0000-0001-7892-3460
}

Recibido - Received - Recebido: 24 / 07 / 2020 Corregido - Revised - Revisado: 15 / 09 / 2020 Aceptado - Accepted - Aprovado: 18 / 09 / 2020

DOI: https://doi.org/10.22458/ie.v22iespecial.3218

URL: https://revistas.uned.ac.cr/index.php/innovaciones/article/view/3218

\begin{abstract}
Resumen: En contextos de pandemia y la sociedad del conocimiento, se hace evidente y exigente la educación virtual relacionada al uso de Tecnologías de Información y Comunicación (TIC). La investigación busca describir entonces el problema social tanto de docentes como de estudiantes en tiempos turbulentos que restringen la educación virtual en las universidades peruanas que a la vez presentan múltiples problemas sociales. Empleándose el método hipotético-deductivo se utilizaron encuestas on line de 2 universidades de gestión estatal y 2 universidades de gestión privada ubicadas en la ciudad de Lima, asimismo, se analizó 4 universidades de gestión estatal y 4 universidades de gestión privada ubicadas en provincias, con un universo poblacional de 260 estudiantes. Se concluye que la educación virtual en las universidades peruanas está sujeta a fracasar debido a la falta de ciertas habilidades de los que imparten conocimiento y a la falta de acceso a internet por los estudiantes provenientes de los espacios periféricos que representan a la mayoría excluida de un país con serias deficiencias económicas, sociales y políticas.
\end{abstract}

Palabras clave: Educación superior, problema social, tecnología de la información, universidad pública, pandemia, COVID-19 


\begin{abstract}
In pandemic and information society contexts, virtual education using information and communication technologies (ICT) becomes evident and urging. The research then seeks to describe both teachers' and students' social problem in turbulent times that restrict virtual education in Peruvian universities that simultaneously present multiple social problems. It was carried out with the help of the hypotheticaldeductive method, online surveys of two state-run universities and two privately-run universities located in the city of Lima. In like manner, four state-run universities and four privately-run universities located in the provinces were analyzed, with a population universe of 260 students. To conclude, that virtual education in Peruvian universities is subject to failure due to the lack of certain skills of those who impart knowledge and the lack of internet access by students from peripheral spaces representing the excluded majority of a country with serious economic, social and political deficiencies.
\end{abstract}

Key Words: Higher Education, social issue, information technologies, public university, pandemic, COVID-19

\begin{abstract}
Resumo: Em contextos de pandemias e da sociedade do conhecimento, a educação virtual relacionada ao uso das Tecnologias da Informação e Comunicação (TIC) torna-se evidente e exigente. A pesquisa procura então descrever o problema social tanto dos professores quanto dos estudantes em tempos turbulentos que restringem a educação virtual nas universidades peruanas que, ao mesmo tempo, apresentam múltiplos problemas sociais. Utilizando o método hipotético-educativo, foram realizadas pesquisas on-line em duas universidades estatais e duas universidades privadas localizadas na cidade de Lima. Da mesma forma, foram analisadas quatro universidades estatais e quatro universidades privadas localizadas nas províncias, com uma população de 260 estudantes. Conclui-se que a educação virtual nas universidades peruanas está sujeita ao fracasso devido à falta de certas habilidades daqueles que transmitem conhecimentos e à falta de acesso à Internet por parte dos estudantes provenientes dos espaços periféricos que representam a maioria excluída de um país com sérias deficiências econômicas, sociais e políticas.
\end{abstract}

Palavras-chave: educação superior, problema social, tecnologia da informação, universidade pública, pandemia, COVID-19

\title{
INTRODUCCIÓN
}

Existe una multiplicidad de problemas sociales que convulsionan la vida cotidiana de las personas y que alteran el funcionamiento de las instituciones sociales como; las actitudes discriminatorias, los hechos de violencia, la desigualdad social y sus consecuencias, las conductas adictivas, la ausencia de conciencia social en los transeúntes y en los conductores, son algunas de las cuestiones que, cada vez más, están presentes en la sociedad y en el acontecer de la vida social e institucional (Belvedere, lardelevsky, Isod y Serulnicoff, 2000; Ocampo y Valencia, 2019; Ponce, Pérez y Hernández, 2016). Si el contexto hubiese sido distinto, se diría que los mismos problemas sociales que aquejan al mundo, a una región o nación, pueden ser tratados en el proceso educativo desarrollado en una institución escolar, lo que posibilitaría concebir una educación integral del alumnado, sustentada en nuevos conocimientos, actitudes y valores, que le permitirán enfrentarlos y erradicarlos (Rodríguez y Rey, 2017).

No obstante, hoy se vive en tiempos de cambios, crisis e incertidumbres nublados bajo el espectro de una enfermedad desconocida denominada virus Corona 2019 (COVID-19) causada por el coronavirus 2 del síndrome respiratorio agudo severo (SARS-CoV-2), considerado como una emergencia de salud pública de preocupación internacional. Hasta ahora, todavía hay controversias sobre la fuente del virus y su huésped intermedio (Li, Yang y Ren, 2020; Ling, 2020; Kampf, Todt, Pfaender y Steinmann, 2020). Lo precedente, ha llevado a la educación a una aparente virtualización dentro de una modalidad de aprendizaje en la que el estudiante tiene que ser más activo y cooperativo en el proceso (Sánchez, 2020; Canaza-Choque y Huanca-Arohuanca, 2018; Huanca-Arohuanca, 2020) así como el docente que está forzado a utilizar herramientas virtuales aunque le resulte difícil.

En el caso concreto del problema de la educación en el Perú, se considera desde un inicio que este es social, económico y político, antes que pedagógico y didáctico, refrendado empíricamente en los contenidos de los lineamientos del Proyecto Educativo Nacional (PEN) y el Proyecto Curricular Regional (PCR), pero que resultan para el Estado peruano sólo clichés declarativos y decorativos; en cuanto, para su implementación al parecer no se ha asignado los presupuestos económicos necesarios y suficientes (Supo, 
2015). Lo anterior se sujeta al bagaje teórico de los conocedores como: Freire (1985; 1990), Bourdieu (1984), Bourdieu y Passeron (1970) y Bauman (2013) respecto a la naturaleza del problema social de la educación.

Por otra parte, la sociedad del conocimiento y la información que muy de cerca ha sido trabajado por Castells (2006), Bauman (2003), Wallerstein (1979; 2005; 2007) y Morín (1994), refieren que la sociedad está en un contexto cambiante de carácter digital que exige de las sociedades el manejo y el dominio de las TIC, porque la sociedad flexible de la modernidad líquida que actúa como un sistema se vuelve cada vez más difícil y complejo. Por ello, es necesario adoptar la perspectiva holística, integral, transdisciplinaria y crítica.

A partir de los preceptos teóricos construidos por los sociólogos arriba mencionados, la realidad social se comporta empíricamente cambiante, más aun, en momentos de la pandemia provocado por el virus de la COVID-19 (Han, Lin, Jin y You, 2020), debido a que, la información digital se ha convertido en una exigencia para el desarrollo de las sociedades y sobre todo para la educación. Por ello, las instancias académicas pertinentes, no deben dejar de lado los problemas sociales y las necesidades de transformación, ya que están comprometidos con la renovación pedagógica de la época y la construcción de la libertad, interés, espontaneidad, creatividad, experimentación, descubrimiento, expresión, autonomía y colectividad (Pardo, 2005) en toda la especie humana.

La educación se constituye como innovadora-crítica y reflexiva a partir de los aprendizajes significativos (Ausubel, 1983) y de implementación de currículos flexibles de competencia. En el caso de la educación superior universitaria, los estudiantes dejan de ser analfabetos clásicos del ayer, hoy son analfabetos contemporáneos. En esa perspectiva, los analfabetos del siglo XXI no serán aquellos que no sepan leer y escribir, sino aquellos que no sepan aprender y desaprender-reaprender (Toffler, 1979) a partir de la información digital. Entonces, se puede especular que estamos ante un contexto de sociedad informacional cuyas características China, Japón, Corea y otras sociedades (potencias mundiales emergentes) han construido su desarrollo.

Sin embargo, en el Perú sólo se ha ensayado imitaciones de otros contextos más relacionados a los aspectos pedagógicos y didácticos. Quizás como decía (Mariátegui, 1928; 1994) la realidad social vinculante a la educación, nunca brote la enseñanza genuina. Lo que sí queda claro es que se ha descuidado los aspectos esenciales como la alimentación y los recursos necesarios para la formación de los educandos, asimismo, se ha dejado de lado la infraestructura adecuada, los medios e instrumentos necesarios, las remuneraciones dignas a la función docente que es necesaria para su formación de calidad. Lo mismo ocurre en la educación superior universitaria, sobre todo en la educación estatal, donde no necesariamente la infraestructura educativa garantiza la calidad y reúne los estándares de normas internacionales exigidos por los organismos competentes.

Por consiguiente, la investigación busca describir el problema social tanto de docentes como estudiantes en tiempos turbulentos que restringen la educación virtual en las universidades peruanas, sabiendo que existen múltiples problemas sociales que impiden el desarrollo o el progreso (Rodríguez y Rey, 2017) de la mayoría de los pueblos, más aun cuanto se enfrentan a un patógeno planetario desconocido que ha pulverizado una serie de susceptibilidades que sumado a ello, tiene el desafío de habitar la peor situación de abandono por el Estado peruano en cuanto se refiere a temas de educación superior. 
Para el constructo de la epistemología de la investigación científica, se utilizó el método de análisis hipotético-deductivo y el método estadístico descriptivo; empleando tablas de distribución de frecuencias por medio de la prueba de chi-cuadrada para verificar la posible relación entre la procedencia de los estudiantes y el acceso que tienen al internet. Además, para el recojo de datos se usó la técnica de la encuesta, cuyo instrumento fue un cuestionario digital vía internet; el cual contó con 5 dimensiones como el acceso a internet, tipo de universidad, procedencia de estudiantes, cobertura y soportabilidad del acceso a la tecnología, así como capacidad de manejo de las TIC.

El instrumento fue validado mediante juicio de expertos con un valor de $85 \%$ de validez y una prueba piloto con una muestra inicial de 52 individuos ( $20 \%$ de la muestra total) que se obtuvo un valor de alpha de Crombach de 0.869 indicando un alto grado de confiabilidad. La muestra estuvo conformada por estudiantes de 12 universidades (02 estatales y 02 privadas) ubicadas en la capital de la república peruana (ciudad de Lima) y 08 universidades (04 estatales y 04 privadas) ubicadas en provincias.

Asimismo, se empleó 260 encuestas accedidas vía virtual por los estudiantes de las universidades participantes de forma probabilística aleatorio-simple por conveniencia, que consiste en la elección por métodos no aleatorios de una muestra cuyas características sean similares a las de la población objetivo (Casal y Mateu, 2003). En esa perspectiva, no se consideró una fórmula estadística. Para mayor detalle de la muestra se tiene la tabla 1.

TABLA 1

Muestra de universidades y población participante

\begin{tabular}{|c|c|c|c|c|c|c|c|c|}
\hline \multirow[b]{2}{*}{$\mathbf{N}^{\circ}$} & \multirow[b]{2}{*}{ Participación de Universidades } & \multicolumn{5}{|c|}{ Características } & \multirow[b]{2}{*}{ Total } & \multirow[b]{2}{*}{$\%$} \\
\hline & & U.E. & U.P. & C.L. & Pro. & $\begin{array}{c}\text { Est. } \\
\text { Part. }\end{array}$ & & \\
\hline 01 & U.N. "Mayor de San Marcos" & 01 & & 01 & & 40 & 40 & 15,41 \\
\hline 02 & U.N. "de Ingeniería" & 01 & & 01 & & 20 & 20 & 07,69 \\
\hline 03 & Pontificia Univers. (PUCP) & 01 & & 01 & & 20 & 20 & 07,69 \\
\hline 04 & Universidad P. “Cayetano H.” & 01 & & 01 & & 20 & 20 & 07,69 \\
\hline 05 & U.N. de Trujillo & 01 & & & 01 & 20 & 20 & 07,69 \\
\hline 06 & U.N. del Centro del Perú & 01 & & & 01 & 20 & 20 & 07,69 \\
\hline 07 & U.N. "San Agustín" Arequipa & 01 & & & 01 & 20 & 20 & 07,69 \\
\hline 08 & U.N. del Altiplano de Puno & 01 & & & 01 & 20 & 20 & 07,69 \\
\hline 09 & Universidad de Huánuco & & 01 & & 01 & 20 & 20 & 07,69 \\
\hline 10 & U. Católica Santa María & & 01 & & 01 & 20 & 20 & 07,69 \\
\hline 11 & U. Andina del Cusco & & 01 & & 01 & 20 & 20 & 07,69 \\
\hline 12 & U. Privada de Tacna & & 01 & & 01 & 20 & 20 & 07,69 \\
\hline \multicolumn{2}{|c|}{ TOTAL } & 08 & 04 & 04 & 08 & 260 & 260 & 100,00 \\
\hline \multicolumn{2}{|c|}{ PORCENTAJE DE PART. UNIV. } & 67 & 33 & 33 & 67 & & 100 & \\
\hline
\end{tabular}

Leyenda:

U.E. : : Universidad Estatal

U.P. : Universidad Privada

C.L. : Ciudad de Lima

Pro. : Provincias (Perú profundo)

Est. Part. : Estudiantes participantes

Fuente: Elaboración propia (2020). 
Para acrecentar y reforzar la investigación en la parte metodológica, se utilizaron las fuentes y bases de datos Scopus, SciELO, Eric, Redalyc, Dialnet, Latindex, Doaj y OEl. Las mismas, que se sostienen en un conjunto de inferencias que se realizaron a partir de la búsqueda, recuperación, análisis, crítica, reflexión e interpretación de datos obtenidos y registrados (Huanca-Arohuanca y Canaza-Choque, 2019; Huanca-Arohuanca, 2019; Huanca-Arohuanca, Sucari, Moriano y Sapana-Valdivia, 2019; Franklin A. Canaza-Choque y Huanca-Arohuanca, 2019; Huanca-Arohuanca, Canaza-Choque, Escobar-Mamani, y Ruelas, 2020).

\section{RESULTADOS}

\section{Acceso a internet por la comunidad universitaria}

Los resultados como evidencias empíricas expresados en la tabla 2, responden a la interrogante formulada: ¿Tiene usted en su condición de estudiante universitario acceso a internet para desarrollar clases virtuales durante la pandemia del COVID-19? Considerando la participación de estudiantes de universidades de gestión pública y privada, ellos refieren que un 55\% sí cuentan con acceso a internet y el $45 \%$ no cuentan con acceso a internet. Sin embargo, considerando la participación sólo de estudiantes de universidades de gestión pública (estatales) ubicadas en la ciudad de Lima y provincias, según los resultados de la tabla 3 , el mayor porcentaje que equivale al $68 \%$ refiere que no tiene acceso a internet y solo un $32 \%$ afirma que tiene acceso a internet.

TABLA 2

Acceso a internet de carácter general

\begin{tabular}{cllll}
\hline \multirow{2}{*}{$\mathbf{N}^{\circ}$} & \multicolumn{1}{c}{ Universidades } & \multicolumn{2}{c}{ Grado acceso } & Total \\
\hline 01 & U.N. "Mayor de San Marcos" & 11 & 29 & 40 \\
02 & U.N. "de Ingeniería" & 08 & 12 & 20 \\
03 & Pontificia Univers. (PUCP) & 18 & 02 & 20 \\
04 & Universidad P. “Cayetano H." & 17 & 03 & 20 \\
05 & U.N. de Trujillo & 08 & 12 & 20 \\
06 & U.N. del Centro del Perú & 07 & 13 & 20 \\
07 & U.N. "San Agustín" Arequipa & 06 & 14 & 20 \\
08 & U.N. del Altiplano de Puno & 05 & 15 & 20 \\
09 & Universidad de Huánuco & 15 & 05 & 20 \\
10 & U. Católica Santa María & 16 & 04 & 20 \\
11 & U. Andina del Cusco & 17 & 03 & 20 \\
12 & U. Privada de Tacna & 14 & 06 & 20 \\
\hline TOTAL & & 142 & 118 & 260 \\
PORCENTAJE & 55 & 45 & 100 \\
\hline
\end{tabular}

Fuente: Elaboración propia (2020). 
Acceso a internet de universidades estatales

\begin{tabular}{cllll} 
& & \multicolumn{2}{c}{ Grado acceso } & Tniversidades \\
$\mathbf{N}^{\circ}$ & & Si & No & Total \\
\hline 01 & U.N. "Mayor de San Marcos" & 11 & 29 & 40 \\
02 & U.N. "de Ingeniería" & 08 & 12 & 20 \\
03 & U.N. de Trujillo & 08 & 12 & 20 \\
04 & U.N. del Centro del Perú & 07 & 13 & 20 \\
05 & U.N. "San Agustín" Arequipa & 06 & 14 & 20 \\
06 & U.N. del Altiplano de Puno & 05 & 15 & 20 \\
\hline TOTAL & & 45 & 95 & 140 \\
PORCENTAJE & 32 & 68 & 100 \\
\hline
\end{tabular}

Fuente: Elaboración propia (2020).

Los recursos digitales y mediáticos han adquirido una relevancia fundamental en el contexto actual y se han establecido como elementos esenciales de la sociedad, ya no solo como facilitadores de la comunicación y de las relaciones interpersonales, sino también como factores clave en la formación del individuo. Por ello, los resultados anteriores evidencian que el estudiante, con la edad, va adquiriendo más consciencia sobre la utilidad de los recursos mediáticos y digitales para fines académicos, dejando de utilizarlos como meros instrumentos de entretenimiento o socialización para comenzar a utilizarlos como herramientas educativas (Bonilla-del-Río, Diego-Mantecón y Lena-Acebo, 2018).

Ahora, en relación con la participación de estudiantes de universidades privadas ubicadas en la capital de la república (ciudad de Lima) y provincias, refieren que $81 \%$ tienen acceso a internet y sólo el $19 \%$ no tiene acceso a esta tecnología, conforme se puede apreciar en la tabla 4.

TABLA 4

Acceso a internet de universidades privadas

\begin{tabular}{|c|c|c|c|c|}
\hline \multirow{2}{*}{$\mathbf{N}^{\circ}$} & \multirow{2}{*}{ UNIVERSIDADES } & \multicolumn{2}{|c|}{ Grado acceso } & \multirow{2}{*}{ Tota } \\
\hline & & & & \\
\hline 01 & Pontificia Univers. (PUCP) & 18 & 02 & 20 \\
\hline 02 & Universidad P. “Cayetano H.” & 17 & 03 & 20 \\
\hline 03 & Universidad de Huánuco & 15 & 05 & 20 \\
\hline 04 & U. Católica Santa María & 16 & 04 & 20 \\
\hline 05 & U. Andina del Cusco & 17 & 03 & 20 \\
\hline 06 & U. Privada de Tacna & 14 & 06 & 20 \\
\hline \multicolumn{2}{|c|}{ TOTAL } & 97 & 23 & 120 \\
\hline \multicolumn{2}{|c|}{ PORCENTAJE } & 81 & 19 & 100 \\
\hline
\end{tabular}

Fuente: Elaboración propia (2020).

Los resultados develan la gran brecha socioacadémica existente en las universidades peruanas a partir del imperio económico. Retomando la idea de teóricos como Marx y los académicos de la escuela de Frankfurt como Marcuse, la humanidad no ha superado el clasismo de que solo un minúsculo grupo debe poseer educación, mientras que la totalidad no puede tener acceso. Hoy se presenta la misma situación. Los que tienen mejores ingresos económicos estudiarán a través de las aulas virtuales y los que no tienen esos ingresos se quedarán bajo el vaivén de lo que pueda hacer el Estado y las universidades públicas. 
En ese punto, aparece como central el habitus, no como un concepto abstracto, sino como un puente que liga la estructura y la agencia al constituir un sistema abierto de experiencias (Pla, 2017) podrían acercar la diferencia de clases en la educación. En ese accionar, al parecer el Estado se ha aislado de algunos estudiantes con niveles de pobreza, que no tiene lugar como un Estado social sino más bien tienen una característica individual interconectada con la indigencia. Porque el problema de la pobreza y la constitución de una clase, y lo que dicen al respecto los/las niños/niñas y los grupos juveniles de un grupo social que se identifica como pobre (Peña y Toledo, 2017).

\section{Procedencia de estudiantes}

La procedencia de estudiantes tanto de universidades públicas como de privadas se encuentran en la ciudad de Lima y en las provincias subalternas. Para el caso de la investigación, la evidencia empírica sostiene que 153 estudiantes que representan el 59\%, proceden de provincias. Mientras que 107 estudiantes que representan el $41 \%$ tienen su residencia establecida en las principales ciudades donde están ubicadas esas universidades. De tal manera que los resultados se pueden apreciar en la tabla 5.

TABLA 5

Procedencia de estudiantes

\begin{tabular}{|c|c|c|c|c|}
\hline \multirow{2}{*}{$\mathbf{N}^{\circ}$} & \multirow{2}{*}{ Universidades } & \multicolumn{2}{|c|}{ Grado de procedencia } & \multirow{2}{*}{ Total } \\
\hline & & Ciud. & Prov. & \\
\hline 01 & U.N. "Mayor de San Marcos" & 09 & 31 & 40 \\
\hline 02 & U.N. "de Ingeniería” & 07 & 13 & 20 \\
\hline 03 & Pontificia Univers. (PUCP) & 13 & 07 & 20 \\
\hline 04 & Universidad P. “Cayetano H.” & 11 & 09 & 20 \\
\hline 05 & U.N. de Trujillo & 05 & 15 & 20 \\
\hline 06 & U.N. del Centro del Perú & 06 & 14 & 20 \\
\hline 07 & U.N. “San Agustín" Arequipa & 08 & 12 & 20 \\
\hline 08 & U.N. del Altiplano de Puno & 04 & 16 & 20 \\
\hline 09 & Universidad de Huánuco & 11 & 09 & 20 \\
\hline 10 & U. Católica Santa María & 12 & 08 & 20 \\
\hline 11 & U. Andina del Cusco & 11 & 09 & 20 \\
\hline 12 & U. Privada de Tacna & 10 & 10 & 20 \\
\hline \multicolumn{2}{|c|}{ TOTAL } & 107 & 153 & 260 \\
\hline \multicolumn{2}{|c|}{ PORCENTAJE } & 41 & 59 & 100 \\
\hline \multicolumn{5}{|c|}{ Leyenda: } \\
\hline Ciud. & : Son de la ciudad & & & \\
\hline Prov. & : Proceden de provincias & & & \\
\hline
\end{tabular}

Fuente: Elaboración propia (2020).

En el contexto del COVID-19, el abordaje de las identidades en los estudiantes universitarios se va diluyendo, así como la pérdida de la temporalidad, los reajustes y las nuevas adquisiciones inestables (Busso, 2017) que se van generando a partir de la interacción virtual. Por otro lado, esos estudiantes inmersos en los medios de comunicación van perdiendo la procedencia original, la visión amable y optimista del fenómeno del cual son parte (Merola y Barbosa, 2017). 


\section{Relación entre procedencia y acceso a internet de estudiantes}

El vínculo que presenta la procedencia y el acceso a internet por los estudiantes de las universidades en mención se encuentra en la tabla 6. Para ello, fue necesario contrastar la siguiente hipótesis estadística:

$\mathrm{H}_{0}$ : No hay relación significativa entre procedencia y acceso a internet

$\mathrm{H}_{1}$ : Sí hay relación significativa entre procedencia y acceso a internet

TABLA 6

Contingencia entre procedencia y acceso a internet

\begin{tabular}{llccc} 
& & \multicolumn{2}{c}{ Acceso a Internet } & Total \\
& & Si & No & \\
\multirow{3}{*}{ Procedencia } & Ciudad & $84(59,2 \%)$ & $23(19,5 \%)$ & $107(41,2 \%)$ \\
& Provincias & $58(40,8 \%)$ & $95(80,5 \%)$ & $153(58,8 \%)$ \\
& Total & $142(100 \%)$ & $118(100 \%)$ & $260(100 \%)$ \\
\hline & & Fuente: Elaboración propia (2020).
\end{tabular}

Para los cálculos correspondientes se empleó el nivel de significancia del $5 \%$ y el test de la chi-cuadrada; ya que se trata de variables cualitativas, pues, los cálculos otorgan un valor de 41,86 para la chi-cuadrada, con un $p$-value de 0.009 , ello quiere decir que sí existe una relación altamente significativa entre el lugar de procedencia y el acceso que tienen a internet, los estudiantes universitarios que provienen de las provincias son los que menos posibilidades tienen frente a los que están en la ciudad.

Hace algunos años atrás, la brecha digital de género referida al acceso a Internet se había reducido, en especial, desde la popularización de la tecnología móvil, a pesar de que los estudios advertían que la persistencia de las desigualdades de género en el uso de las tecnologías puede perpetuarse en otras dimensiones como la experiencia, habilidad, autonomía y tipos de uso (Vico-Bosch y Rebollo-Catalán, 2019). Hoy el problema renace con la brecha digital del acceso a internet para sustentar las aulas virtuales que las universidades han abierto.

\section{Cobertura y soportabilidad del acceso a la tecnología en universidades públicas y privadas}

La cobertura y soportabilidad del acceso a la tecnología (internet) en las universidades de gestión pública y privada, ubicadas geográficamente en la capital de la república y en provincias, obedece a la siguiente interrogante: ¿cree usted que la capacidad de cobertura y soportabilidad del acceso a la tecnología en su Universidad es suficiente para desarrollar sesiones de educación virtual? Los estudiantes de universidades de gestión pública respondieron en un 70\%, que no existe suficiente capacidad de cobertura y soportabilidad del acceso a la tecnología de internet para desarrollar sesiones de educación virtual (ver tabla 7); mientras que, los estudiantes de universidades de gestión privada respondieron en un $69 \%$, que sus universidades sí tienen la capacidad suficiente de cobertura y soportabilidad para desarrollar sesiones de educación virtual, conforme se puede apreciar en la tabla 8. 
TABLA 7

Cobertura y soportabilidad del acceso a internet de universidades públicas

\begin{tabular}{clccc}
$\mathbf{N}^{\circ}$ & $\begin{array}{c}\text { Universidades } \\
\text { de gestón pública }\end{array}$ & $\begin{array}{c}\text { COBERTURA Y SOPORTABILIDAD } \\
\text { Si }\end{array}$ & TOTAL \\
\hline 01 & U.N. "Mayor de San Marcos" & 10 & 30 & 40 \\
02 & U.N. "de Ingeniería" & 07 & 13 & 20 \\
03 & U.N. de Trujillo & 06 & 14 & 20 \\
04 & U.N. del Centro del Perú & 07 & 13 & 20 \\
05 & U.N. "San Agustín” Arequipa & 08 & 12 & 20 \\
06 & U.N. del Altiplano de Puno & 04 & 16 & 20 \\
\hline TOTAL & 42 & 98 & 140 \\
PORCENTAJE & 30 & 70 & 100 \\
\hline
\end{tabular}

Fuente: Elaboración propia (2020).

TABLA 8

Cobertura y soportabilidad de acceso del internet de universidades privadas

\begin{tabular}{clccc}
\hline & N & Universidades de gestión privada & Cobertura y soportabilidad & Total \\
\hline 01 & Pontificia Univers. (PUCP) & Si & No & 20 \\
02 & Universidad P. "Cayetano H." & 19 & 01 & 20 \\
03 & Universidad de Huánuco & 18 & 02 & 20 \\
04 & U. Católica Santa María & 10 & 10 & 20 \\
05 & U. Andina del Cusco & 13 & 07 & 20 \\
06 & U. Privada de Tacna & 12 & 08 & 20 \\
\hline TOTAL & & 11 & 09 & 120 \\
PORCENTAJE & 83 & 37 & 100 \\
\hline
\end{tabular}

Fuente: Elaboración propia (2020).

Los estudiantes del ámbito estatal se encuentran en una desventaja en comparación con los estudiantes de procedencia privada en cuanto a la cobertura y a la soportabilidad. En las universidades del Perú existe desde casi siempre la carencia al acceso a internet, compra o alquiler de licencias, red interna de datos en la organización, equipos de cómputo actualizados, servidores confiables y de alta capacidad de almacenamiento (Cano y Baena, 2017).

\section{Capacidad de manejo de las TIC en las universidades públicas y privadas}

Cuando se pretende apreciar las tablas 9 y 10, las capacidades de manejo de las TIC, la investigación se remite a la siguiente pregunta: ¿Cuánto conoce usted de la capacidad de manejo de las TIC en el entorno a aplicaciones educativas (buen manejo, regular manejo y mal manejo) por parte de los docentes de su Universidad para desarrollar sesiones de educación virtual? Los estudiantes de universidades de gestión pública respondieron en un 38\% (58 estudiantes) que sus docentes se encuentran en la fase regular, seguido de un $36 \%$ (50 estudiantes) de alumnos que respondieron con un bueno y el $26 \%$ (36 estudiantes) respondió que sus profesores tienen un mal manejo de la tecnología. Mientras que los estudiantes de 
universidades de gestión privada respondieron en mayor porcentaje, que la capacidad de manejo de las TIC como aplicativos de carácter académico es bueno, alcanzando un 71\% (85 estudiantes), en cambio un 21\% (26 estudiantes) respondió que es regular y sólo el 08\% (09 estudiantes) respondió que la capacidad de manejo de TIC de los docentes es mala.

TABLA 9

Capacidad de manejo de TIC por docentes de universidades públicas

\begin{tabular}{clcccc} 
& & \multicolumn{2}{c}{ CAPACIDAD DE MANEJO } & \multicolumn{2}{c}{ Total } \\
\hline 01 & Universidades de gestón pública & Uueno & Regular & Malo & \\
02 & U.N. "de Ingeniería" & 12 & 18 & 10 & 40 \\
03 & U.N. de Trujillo & 08 & 06 & 06 & 20 \\
04 & U.N. del Centro del Perú & 06 & 10 & 04 & 20 \\
05 & U.N. "San Agustín" Arequipa & 05 & 11 & 04 & 20 \\
06 & U.N. del Altiplano de Puno & 09 & 06 & 05 & 20 \\
\hline TOTAL & 10 & 03 & 07 & 20 \\
PORCENTAJE & 50 & 54 & 36 & 140 \\
\hline
\end{tabular}

Fuente: Elaboración propia (2020).

TABLA 10

Capacidad de manejo de TIC por docentes de universidades privadas

\begin{tabular}{llcccc} 
& & \multicolumn{2}{c}{ Capacidad de manejo } & Total \\
\hline & Universidades de gestión privada & Bueno & Regular & Malo & \\
\hline 01 & Pontificia Univers. (PUCP) & 18 & 02 & 00 & 20 \\
02 & Universidad P. "Cayetano H." & 19 & 01 & 00 & 20 \\
03 & Universidad de Huánuco & 14 & 04 & 02 & 20 \\
04 & U. Católica Santa María & 13 & 06 & 01 & 20 \\
05 & U. Andina del Cusco & 11 & 06 & 03 & 20 \\
06 & U. Privada de Tacna & 10 & 07 & 03 & 20 \\
\hline TOTAL & & 85 & 26 & 09 & 120 \\
PORCENTAJE & 71 & 21 & 08 & 100 \\
\hline
\end{tabular}

Fuente: Elaboración propia (2020).

La ambivalencia salta a la vista en los contextos de la COVID-19, cuando por alguna extraña razón los docentes de las instituciones estatales manejan en menor número las TIC, No obstante, pasa casi lo contrario con los docentes del ámbito privado, dando entrever que las aulas virtuales no tendrán resultados positivos en lo que va el transcurso académico universitario.

Entonces, a las dificultades habituales observadas en la relación pedagógica se suman ahora un contexto juvenil en el que el uso masivo de TIC relativiza la importancia tanto de la figura del docente como de los modos de aprendizaje estándar. Se requieren nuevas formas de relación educativa, cualificar al profesorado, renovar los cauces de enseñanza y la filosofía de trabajo (Plaza, 2018). En definitiva, la creación de nuevos medios de transporte y la revolución de los sistemas de comunicación fueron factores que a su vez contribuyeron al aumento de los bienes de consumo y, por consiguiente, al modelo actual de desarrollo consumista que merece ser reemplazado por uno nuevo (Rosas y Barrios, 2018). 


\section{DISCUSIÓN}

El conocimiento no escapa a ninguna realidad, la virtualización de la sociedad está a la orden del día, no se está lejos de comprar, abastecer y educar utilizando esos medios, lo cierto es que el mundo ya no será como ayer. Se asiste a cambios rápidos y violentos, esa no es una revolución socialista ni comunista es la revolución del conocimiento, resistirse sería salir de ese proceso, por ello, es necesario entrar y alinearse a esos desafíos, y los maestros tienen que jugar un rol en ello, a pesar de que existan muchas dificultades, como la falta de capacitación, el acceso a internet limitado, la infraestructura y las plataformas que caen porque sus arquitecturas adolecen de innovaciones; la disyuntiva es, si no se entra a ese entorno, el precio que se pagará será alto. Lo cierto es que, la educación virtual es una realidad que no podemos evitar (Castells, 2006; Bauman, 2003; Wallerstein, 1979; Morin, 1994).

Sin embargo, la realidad social en contextos de la COVID-19 de los estudiantes universitarios en la sociedad peruana es muy diferenciado. Una de las principales diferencias es el contexto; las universidades donde estudian están ubicadas unas en la capital de la república y otras en provincias. Otra de las diferencias se acentúa en la naturaleza de las universidades ya que estas pueden son nacionales o privadas, con fines y sin fines de lucro. En ese criterio, la posibilidad de implementar la enseñanza no presencial de carácter virtual, puede, como no, tener éxito. Dicho lo anterior, las posibilidades de tener mayor ventaja al acceso de las TIC está en aquellos estudiantes que pertenecen a universidades privadas. No siendo lo mismo para los estudiantes de gestión estatal, que en gran mayoría son provincianos pertenecientes a la subalternidad.

Esa realidad social, permite manifestar que las estrategias académicas de enseñanza virtual implementados por las autoridades de universidades estatales y privadas a nivel nacional, no garantizarán el aprendizaje efectivo de los miles de estudiantes con ascendencia provinciana e indígena. En definitiva, la educación virtual no resulta aplicable para desarrollar los aprendizajes esperados en la comunidad universitaria nacional, porque internet a alta gama en los estudiantes de provincia, sigue siendo uno de los desafíos que tendrán que ser resueltos por los agentes educativos contemplados en: los gobiernos, universidades, alumnos, profesores y la sociedad civil.

\section{SÍNTESIS Y REFLEXIONES FINALES}

Evidentemente, la aldea global está atrapada en una pandemia mundial generadora de una serie de variables negativas en las sociedades del tercer mundo. En ese empuje, solo la buena interacción alcanzada en la sociedad civilizada y un Estado fuerte podrá resistir el aislamiento social que vulnera una serie de derechos. Asimismo, solo una educación democrática en todos los niveles podrá afrontar el futuro apocalíptico al cual se expone la humanidad.

Ahora bien, el estudio concluye que el $55 \%$ de estudiantes cuentan con acceso al internet y el $45 \%$ no. Por otro lado, un $38 \%$ de estudiantes de universidades de gestión pública consideran que sus docentes se encuentran en la fase regular, Mientras que el $71 \%$ de los estudiantes de universidades de gestión privada consideran que la capacidad de manejo de las TIC en sus docentes es bueno, lo que pone en ventaja a los últimos con respecto a los estudiantes de provincias.

En torno a los estudiantes de universidades de gestión pública, estos sustentan en un $70 \%$ que no existe suficiente capacidad de cobertura y soportabilidad del acceso a la tecnología de internet para desarrollar sesiones de educación virtual. No obstante, los estudiantes de universidades de gestión privada respondieron en un $6 \%$ que sus universidades sí tienen la capacidad suficiente de cobertura y soportabilidad para desarrollar sesiones de educación virtual. Y otra vez, los actores estudiantiles de carácter privado se anteponen a los que vienen de la periferia. 
De modo que la existencia de una diferenciación social en los estudiantes de universidades de gestión estatal y privada, según su ubicación, retoma el viejo determinismo económico que consistía en: los que tienen dinero podrán estudiar en universidades privadas y los que no, simplemente se quedarían en las instituciones estatales carentes de calidad. Hoy se hace visible una vez más la diferencia de clases, los estudiantes provincianos no tienen acceso a internet, lo que significa que no recibirán las clases virtuales, pero los que pueden acceder a internet ilimitado si podrán realizar esas clases virtuales porque son de la capital o alguna universidad privada con buena presencia. En conclusión, la diferenciación es un problema social de la educación porque presenta ciertas restricciones que no garantizan el desarrollo de la educación universitaria en el Perú multiverso.

\section{REFERENCIAS}

Ausubel, D. (1983). Teoría del aprendizaje significativo. Fascículos de CEIF, 1, 1-10.

Bauman, Z. (2003). Modernidad líquida . México: Fondo de Cultura Económica.

Bauman, Z. (2013). Sobre la educación en un mundo líquido. Conversaciones con Ricardo Mazzeo. Ediciones Paidós.

Belvedere, C., lardelevsky, A., Isod, L., \& Serulnicoff, A. (2000). Los problemas sociales y la escuela. Desigualdad y vulnerabilidad social (Vol. 14, Issue 2). Ministerio de Educación, Ciencia y Tecnología. https://doi.org/10.4067/S1726-569X2008000200002

Bonilla-del-Río, M., Diego-Mantecón, J. M., \& Lena-Acebo, F. J. (2018). Estudiantes Universitarios: prosumidores de recursos digitales y mediáticos en la era de internet. Aula Abierta, 47(3), 319-326. https://doi.org/10.17811/rifie.47.3.2018.319-326

Bourdieu, P. (1984). Homo academicus. Siglo XXI.

Bourdieu, P., \& Passeron, J. (1970). La reproducción. Elementos para una teoría del sistema de enseñanza. Fontamara.

Busso, M. P. (2017). Los emigrantes argentinos y la construcción de identidades nacionales en soportes de Internet. Observatorio (OBS*) Journal, 61-89.

Canaza-Choque, Franklin A., \& Huanca-Arohuanca, J. W. (2019). Disputas por el oro azul: gobernanza hídrica y salud pública. Rev. Salud Pública, 21(5), 1-7. https://doi.org/10.15446/rsap.V21n5.79646

Canaza-Choque, Franklin Américo, \& Huanca-Arohuanca, J. W. (2018). Perú 2018: hacia una Educación Intercultural Bilingüe sentipensante. Sciendo, 21(4), 515-522. https://doi.org/10.17268/ sciendo.2018.058

Cano, J. A., \& Baena, J. J. (2017). Limitaciones en el uso y apropiación de tecnologías de información y comunicación para la negociación internacional en empresas colombianas. Observatorio (OBS*) Journa, 11, 111-133. http://www.scielo.mec.pt/pdf/obs/v11n1/v11n1a08.pdf

Casal, J., \& Mateu, E. (2003). Tipos de Muestreo. Rev. Epidem. Med. Prev, 1, 3-7. https://www.coursehero. com/file/9890875/TiposMuestreo1/

Castells, M. (2006). La sociedad red: Una visión Global. Alianza Editorial.

Freire, P. (1985). Pedagogía del oprimido. Siglo XXI.

Freire, P. (1990). La naturaleza política de la educación. Cultura, poder y liberación. Ediciones Paidós.

Han, Q., Lin, Q., Jin, S., \& You, L. (2020). Coronavirus 2019-nCoV: A brief perspective from the front line. Journal of Infection, 80(4), 373-377. https://doi.org/10.1016/j.jinf.2020.02.010 
Huanca-Arohuanca, J. W. (2019). El discurso filosófico y la violencia política en la Nación Aymara Ácora [Universidad Nacional del Altiplano]. http://repositorio.unap.edu.pe/bitstream/handle/ UNAP/12758/Huanca_Arohuanca_Jesús_Wiliam.pdf?sequence=1\&isAllowed=y

Huanca-Arohuanca, J. W. (2020). Contrahegemonía y la lucha por la educación en el sur del Perú. Editorial Académica Española.

Huanca-Arohuanca, J. W., \& Canaza-Choque, F. A. (2019). Puno: Educación rural y pensamiento crítico. Hacia una educación inclusiva. Revista Helios, 3(1), 97-108. https://doi.org/10.22497/Helios.31.3106

Huanca-Arohuanca, J. W., Canaza-Choque, F. A., Escobar-Mamani, F., \& Ruelas, D. (2020). En defensa del pluralismo latinoamericano: las esferas de la justicia y la igualdad compleja en Michael Walzer. Un dilema pendiente por atender. Revista Chakiñan, 11, 92-103. https://doi.org/10.37135/chk.002.11.07

Huanca-Arohuanca, J. W., Sucari, W., Moriano, J., \& Sapana-Valdivia, N. (2019). Valoración caósmica de la globalización en los sistemas educativos: una aproximación crítica desde la filosofía decolonial. Revista Innova Educación, 1(4), 411-421. https://doi.org/10.35622/j.rie.2019.04.001

Kampf, G., Todt, D., Pfaender, S., \& Steinmann, E. (2020). Persistence of coronaviruses on inanimate surfaces and their inactivation with biocidal agents. Journal of Hospital Infection, 104(3), 246-251. https://doi.org/10.1016/j.jhin.2020.01.022

Li, C., Yang, Y., \& Ren, L. (2020). Análisis de la evolución genética del nuevo coronavirus y coronavirus de 2019 de otras especies. Infección, Genética y Evolución, 28. https://doi.org/10.1016/j. meegid.2020.104285

Ling, C. (2020). La medicina tradicional china es un recurso para el descubrimiento de fármacos contra el nuevo coronavirus 2019 (SARS-CoV-2). Revista de Medicina Integrativa, 18(2), 87-88. https://doi. org/10.1016/j.joim.2020.02.004

Mariátegui, J. C. (1928). El proceso de la Instrucción Pública. In 7 Ensayos de Interpretación de la Realidad Peruana (p. 1928). Ediciones Mercurio.

Mariátegui, J. C. (1994). Temas de Educación. In Mariátegui Total (p. 1994). Ediciones Mercurio.

Merola, J. S., \& Barbosa, M. V. (2017). Representar la migración juvenil. Estudio comparativo de sus imaginarios simbólicos en la televisión y en las redes sociales digitales. Revista de Dialectología y Tradiciones Populares, 72(1), 37-42. https://doi.org/10.3989/rdtp.2017.01.001.04

Morin, E. (1994). Introducción al pensamiento complejo. Gedisa.

Ocampo, L. F., \& Valencia, S. (2019). Los problemas sociales relevantes: enfoque interdisciplinar para la enseñanza integrada de las ciencias sociales. Revista de Investigación En Didáctica de Las Ciencias Sociales, 4, 60-75. https://doi.org/10.17398/2531-0968.04.60

Pardo, B. (2005). La Escuela Nueva como corriente pedagógica: principios que necesita la educación actual. UNAM.

Peña, M., \& Toledo, C. (2017). Ser pobre en el Chile Neoliberal: Estudio discursivo en una escuela vulnerable. Revista Latinoamericana de Ciencias Sociales, Niñez y Juventud, 15(1), 207-218. https://doi.org $/ 10.11600 / 1692715 \times .1511225012016$

Pla, J. L. (2017). Trayectorias de clase y percepciones temporales sobre la posición ocupada en la estructura social. Un abordaje multimensional de las clases sociales argentinas (2003-2011). Revista Internacional de Sociología, 75(3), 1-14. https://doi.org/10.3989/ris.2017.75.3.16.05

Plaza, J. (2018). Autoridad docente y Nuevas Tecnologías: cambios, retos y oportunidades. Revista Complutense de Educación, 29(1), 269-285. https://doi.org/10.5209/RCED.52281

Ponce, L., Pérez, R., \& Hernández, R. E. (2016). Problemas sociales de la ciencia en la educación superior para las ciencias agrarias en Cuba. Revista Universidad y Sociedad [Seriada, 8(2), 187-195. http:// scielo.sld.cu/pdf/rus/v8n2/rus24216.pdf 
Rodríguez, O., \& Rey, C. (2017). Los problemas sociales y su contextualización en el proceso educativo escolar: una necesidad actual. Revista Electrónica "Actualidades Investigativas En Educación," 17(2), 1-17. https://doi.org/10.15517/aie.v17i1.28150

Rosas, M. E., \& Barrios, A. (2018). Comunicación de riesgo, cambio climático y crisis ambientales. Chasqui. Revista Latinoamericana de Comunicación, 136, 179-194. https://revistachasqui.org/index. php/chasqui/article/view/3294/2983

Sánchez, J. A. (2020, March 24). Virtualidad: la pandemia que cambió la educación superior para siempre. El Tiempo, 1-6. https://www.eltiempo.com/vida/ciencia/ educacion-virtual-la-pandemia-que-cambio-la-educacion-superior-para-siempre-476390

Supo, F. (2015). El problema de la educación en el Perú. UNEEGV.

Toffler, A. (1979). La tercera ola. Bantam Books.

Vico-Bosch, A., \& Rebollo-Catalán, Á. (2019). El aprendizaje de las mujeres sobre internet y redes sociales: validación y resultados generales de una escala. Educación XX1, 22(1), 375-400. https://doi. org/10.5944/educXX1.21469

Wallerstein, I. (1979). El moderno sistema mundial (Tomo I). Siglo XXI.

Wallerstein, I. (2005). Análisis de sistemas-mundo. Una introducción (2a. edició). Siglo XXI.

Wallerstein, I. (2007). Geopolítica y geocultura. Ensayos sobre el moderno sistema mundial. Kairos. 